\title{
The Application of 4C Training Model in the Operating Room Nurse Training
}

\author{
Feng Zhao, Cui Wang and Ruofeng Yin ${ }^{\text {a }}$ \\ China-Japan Union Hospital of Ji Lin University, Changchun, Jilin, China
}

\begin{abstract}
The operating room is an important place for the treatment of patients undergoing surgery, the quality level of clinical nursing has a direct influence in the therapeutic effect of patients. In the present study, four components instructional design mode (4C training mode) was applied in the training of professional nurses in the operating room, it involved the following aspects: regular skill knowledge analysis, goals setting, as well as the construction and implementation of the project. Targeted training methods and training content were formulated in accordance with the learning ability and knowledge reserve of different professional nurses, and different skills required to master, to fully mobilize the enthusiasm and initiative of nurses, thereby to improve the operation and comprehensive level of nurses in the operation room to achieve good results.
\end{abstract}

\section{Introduction}

Operating room is the most important place for operative treatment of patients and the quality of nursing work within the operating room is also one of the most critical standards measuring the overall medical level and service quality of a hospital. Work in the operating room is not only complicated, but involved with multiple disciplines. Hence, it is required to be provided with a wellqualified nursing team. How to improve the overall quality of nurses in the operating room, rationally utilize limited human resources, sufficiently arouse the enthusiasm of nurses and improve work efficiency and nursing quality of nurses in the operating room is the rigorous problem confronted with by operating room nursing managers.

With the rapid development of contemporary surgery, surgical operations have grown into diversification from the original simplification. In addition to continuous expansion of surgical fields and scopes, new surgical methods, new instruments and new apparatus also keep emerging, which asks operating room nurses (ORNs) to be transformed into those intensive, highly specialized and refining constantly from conventional simple single and crude coordination. In other words, requirements for level of expertise and overall quality of ORNs become increasingly higher. Only well-qualified ORNs are able to coordinate with specialized surgeries that are developing towards superior difficulty and refinement. Considering this, effective training for nurse specialists is particularly important[1]. In this study, the 4C training pattern is applied into operating room nurse specialist training that has achieved preferable effects according to the relevant observations. As for this pattern, it is not only effective in improving operating room nurse specialist training effects, but also beneficial to cultivate modern ORNs highly specialized and versatile. As a result, operating room nursing work complies with the development of surgery and work of ORNs can also be comprehensively and systematically improved. Concerning the associated details, they are described below.

\footnotetext{
${ }^{a}$ Corresponding author : Ruofeng Yin, Email:50703463@qq.com
} 


\section{Information and methods}

\subsection{General information}

160 nurses working in the operating room of our hospital from March 2014 to March 2015 are selected and randomly divided into a control group and a study group by means of random number table. For each group, there are 80 nurses. As for the study group, the $4 \mathrm{C}$ training pattern is adopted to carry out specialized trainings for nurses here; in comparison, the regular operating room specialist training is employed by the control group. Differences in general data such as age, sex, education background and working hours, etc. of nurses from such two groups have no statistical significances during comparisons $(\mathrm{P}>0.05)$, in addition to being comparable.

\subsection{Methods}

\subsubsection{Conventional specialist training}

Dependent on job demands of departments, operating rooms are categorized into 7 specialist groups including general surgery, cardiothoracic surgery, orthopedics, neurosurgery, gynaecology and obstetrics, and urinary surgery, etc.; in which, personnel coordinating with specialized operations take their turns regularly and the corresponding nurse specialists of diverse specialist groups are trained targeted at basic knowledge, fundamental theories and skills that should be mastered by them to cooperate with various specialized operations. In addition, the relevant training contents and modes are uniformly arranged by the head nurse in charge of teaching. After the training, these nurses are also assessed[2].

\subsubsection{C Training pattern}

In line with conventional specialist training methods, 7 specialized groups are allocated with nurse specialists according to practical surgical operation quantities and workload, and each group comprises 1 leader and 9-11 members. To be specific, members consist of fixed team members (2-3) and team members taking turns (6-8). As for the former, they assist the team leader to formulate and implement working plans. Members of every group are allocated proportionally to form a talent pyramid so as to guarantee stable operation and future development of these specialist groups. Regarding skilled team leaders with strong work abilities and senses of responsibilities, they should be nurse-in-charge or more qualified personnel with a certain management capability. The development direction of each specialist group should be organized by the head nurse in charge of teaching and also discussed in detail among fixed members of it. At the same time, the corresponding training plans, goals and elaborate head nurses should be established together with the relevant implementation schemes. Then, according to these schemes, management objectives can be decomposed and become practical in terms of each related post. Nurses of specialist groups are trained so that they have the capability to fulfill the established training goals based on the existing plans and schemes.

\subsubsection{Prospect analysis}

Such a stage can be classified into three aspects. First is the demand analysis on specialized operation cooperation. Nurse specialists are cultivated with an aim to cooperate with demands of various specialized operations in a better manner, and make ORNs able to rapidly and effectively master working skills corresponding to these specialized operations and qualified for specialized operation 
cooperation in rapid development. Considering this, demands of diverse specialized operations should be analyzed elaborately to precisely and comprehensively decompose key points of specialized operation cooperation. Second is feature analysis on training objects. As the subject of learning, training objects are crowd that should be changed. In order to achieve good training effects, education backgrounds and working experiences of them should be mastered in the first place. Then, individualized specialist trainings are formulated corresponding to their different learning and working experiences, etc. In this way, training demands can conform to characteristics and styles of diverse trainees. Thirdly, it is about the analysis on department development demands. Due to the rapid growth of surgery, new techniques and new devices keep emerging for the operating room and the present operating room nursing is also at a stage of rapid development. As for training of ORNs that comply to cooperation needs of rapid surgical operation development and satisfy rational construction demands of department personnel echelon, perfect feasible training plans should be formulated with taking physical truths of these departments, time, costs and staff composition, etc. into consideration.

\subsubsection{Objective setting}

Objective setting is a greatly important link, because objectives are theoretical basis for training plan preparation and can provide directions for training. According to the $4 \mathrm{C}$ training pattern, objectives are divided into two categories. One is the objective that can be repeatedly applied, that is, the content that should be mastered for a long time during practical work. Combining particulars of work in the operating room, it includes the common conventional operation cooperation, as well as use, maintenance and simple troubleshooting of apparatus and equipment in common use. The other is the objective that cannot be applied over and over again. It refers to skills that should be mastered during temporary jobs. In combination with specifics of work in the operating room once more, it consists of operating room management. In detail, it is involved with management methods that should be changed flexibly according to different circumstances at different periods. Besides, it also involves treatment of emergencies and problems arising in the operating room, etc.

\subsubsection{Construction of scheme}

After the fulfillment of objective setting, we duly set about establishing training schemes. In these schemes, prominence should be given to the key points and emphasis is also laid on cultivating specialized abilities of ORN specialists as well as their comprehensive qualities. Thus, the overall nursing level and synthetic abilities in an operating room can be improved. From the perspective of scheme contents, the status quo of training objects should be grasped through self-assessment by trainees and preliminary assessment by specialist groups, so as to prepare scheme contents appropriate to these objects in accordance with their present situations and objective design. During scheme establishment, learning resources and environment of the department are reasonably utilized to carry out multimedia teaching, professional lectures, operation cooperation practice demonstration and interrelated resource learning in a variety of forms and contents. In detail, with regard to the multimedia teaching, knowledge points and cutting-edge technologies of the department are taught by means of multi-media network teaching. Regarding the professional lectures, diseases and the corresponding therapeutic methods, surgical procedures and nursing cooperation, etc. of the department are systematically explained by surgeons and senior nurse specialists. About the operation cooperation practice demonstration, it is not elaborated here. Finally, for interrelated resource learning, self-knowledge is supplemented through books, database resources and network teaching videos. 


\subsubsection{Implementation of scheme}

This section covers three aspects described below. Firstly, a perfect hierarchical management system under the control of head nurses of departments should be set up. Various specialized departments are planned as a whole by the head nurse in charge of teaching; while, the responsibility to implement specialist trainings in concrete should be borne by the specialist group leaders. Such a vertical hierarchical management system is more beneficial to define objectives clearly and improve the abilities of specialist group leaders to play a dominant role, exert all capabilities fully, and actively participate into formulating and supervising trainings for the corresponding nurses in all specialist groups. Secondly, each specialist group leader takes the responsibility to settle and summarize operation techniques and theories that should be mastered by the corresponding department, so as to form an integral knowledge and skill learning system. Furthermore, all kinds of skills and knowledge are made into courseware for seminars and lectures as theoretical trainings on a regular basis. Professional technical operations are performed in a sequence of specialist operation-specialist emergency operation-new technique. Thirdly, technical files should also be established to improve the assessment system of a department. In addition to completing tests arranged and required by the nursing department, contents of assessment are additionally added by the specialist department.

\subsubsection{Evaluation criterion}

The evaluation criterion is constituted by assessment of theoretical knowledge, assessment of operating room nursing manipulation and assessment of professional ability. The manipulation assessment refers to the uniform standard and manipulations are selected randomly to be rated. Both theoretical and manipulation assessments are 100 points, and it is deemed to be qualified if 60 and above points are obtained. With regard to the professional ability assessment, it is carried out by the Comprehensive Quality Evaluation Table of ORNs prepared by the department, covering clinical competency, learning time management ability, solidarity cooperation ability, communication skills, specialty knowledge level and operation coordination ability. Each of them is endowed with 10 points. That graded to be 8 points and above is qualified, while below 8 points is unqualified.

\section{Statistical analysis}

Statistical software SPSS 20.0 is employed to perform statistical analysis on all data. Enumeration data are described by rate and tested by $\chi^{2}$. By contrast, measurement data are accounted by Mean \pm Standard Deviation and verified by t. Besides, those with $\mathrm{P}<0.05$ is considered to be different and have statistical significances.

\section{Results}

Regarding nurses from the study group qualification rate, qualification rates obtained from assessment of theoretical knowledge, assessment of operating room nursing manipulation and assessment of professional ability are respectively $97.5 \%, 92.5 \%$ and $95 \%$, which is prominently higher than $82.5 \%$, $75.0 \%$ and $77.5 \%$ of the control group. Differences comparisons of such two groups are statistically significant $(\mathrm{P}<0.05)$, as shown in Table 1 . 
Table 1. The Compare two groups of nurse training results.

\begin{tabular}{cccc}
\hline Group & $\begin{array}{c}\text { qualification rate of basic } \\
\text { knowledge }\end{array}$ & $\begin{array}{c}\text { Operation qualification } \\
\text { rate }\end{array}$ & $\begin{array}{c}\text { Business capability } \\
\text { assessment }\end{array}$ \\
\hline $\begin{array}{c}\text { Control group } \\
\text { Observation }\end{array}$ & $82.5 \%$ & $75.0 \%$ & $77.5 \%$ \\
group & $97.5 \%$ & $92.5 \%$ & $95.0 \%$ \\
$\chi^{2}$ & & & \\
$\mathrm{p}$ & 5.00 & 4.50 & 5.16 \\
& $\mathrm{P}<0.05$ & $\mathrm{P}<0.05$ & $\mathrm{P}<0.05$ \\
\hline
\end{tabular}

\section{Discussion}

The operating room is an important place where patients are treated. With the continuous development of science and technology and constant improvement of medical level, higher requirements have been put forward by the entire society for operating room nursing. Integrating multiple related disciplines, operating room nursing has higher requirements of nurses. Therefore, trainings for them are of great importance. On one hand, by virtue of such trainings, skill level of them can be improved; on the other hand, guarantees are provided for the improvement of nursing quality in the hospital. How to exploit potentials of nurses in a better manner and make the trainings yield twice the result with half the effort is a subject that all managers should face [3-5].

The 4C training pattern includes regularly decomposing skills that should be mastered by surgery nurses, analyzing skill elements and relevant knowledge, selecting teaching strategy, and assembling training strategy. The team responsible for the project should decompose skills that should be grasped by nurses in the operating room in the first place, mainly covering clinical care, nursing supervision, nursing scientific research and teaching. Then, contents at all levels are decomposed further. To be specific, the clinical care is involved with: basic knowledge of operating room nursing, placement of operative position, anesthesia preparation and cooperation, cooperation with mini-middle-operations in diverse departments[6]; mastering particularities associated with surgical methods, instrument and dressing preparations; comprehending new surgical techniques and key points for cooperation with salvage surgery; fostering emergency rescue capabilities; and, precautions to operating room nursing errors; etc.. In summary, clinical care training is about the repeated application and strengthening of skills during trainings for nurses in diverse departments. In terms of operating room management, nursing scientific research and teaching trainings, they should be conducted combining personal interest and competency on the basis of senior nurses with certain experiences. 4C training pattern applied into nurse specialist trainings should be adopted to prepare targeted training methods and contents according to learning abilities, knowledge reserves and skills required mastering of nurses from diverse departments, so that learning enthusiasm and initiative of them can be sufficiently mobilized and the goal to improve manipulation and comprehensive levels of ORNs is fulfilled. In addition, thanks to the employment of $4 \mathrm{C}$ training pattern, systematicness of operating room nursing is reinforced, which is conducive to improvement of operating room nursing quality and efficiency in the hospital. This study indicates that, in the study group taking advantage of such a 4C training pattern, qualification rates relate to all assessments of nurses are obviously higher than those of nurses from the control group receiving conventional specialist training.

In conclusion, operating room nurse specialist training that adopts the $4 \mathrm{C}$ training pattern can significantly enhance various competencies of nurses, and improve qualities of nurse specialists. Considering such a preferable application effect, it is worth popularization and application.

\section{References}

1. Ying Qiu, Jing Zhang. Study on setting up of training courses of operation room nurse specialists[J]. Chinese Nursing Research 2014, 28(8B) :2859-2862. 
2. Yunmiao Ma, Anling Li, Huimin Zhao. Research status quo of core competence of specialist nurses in operating rooms at home and in abroad[J]. Chinese Nursing Research 2013, 29(3): 10291031.

3. Yingyu Zeng, Xiaodan $\mathrm{Wu}$, Guiying $\mathrm{Xu}$, et al. Correlation study between core competence and fixed specialty in operating room nurses[J]. Journal of Nursing Administration 2014, 14(11):778788.

4. Lianying Li. Specialist in the operating room nurses training mode[J]. Nursing Practice and Research 2013, 10(11):142-143.

5. Feihong Lan. Specialist in the operating room nurses the cultivation of the status quo[J]. Today Nurse 2015, 2:16-18.

6. Yan Ding, Weijing Liu. Investigation on core competency and curriculum design for operatingroom nurse speclalists[J]. Journal of Nursing Seience 2013, 28(22):4-5. 